\title{
Seborrhoeic dermatitis and a herpes zoster infection developed during treatment with adalimumab due to Crohn's disease
}

\author{
Beata Bergler-Czop ${ }^{1}$, Dominika Wcisło-Dziadecka², Karolina Wodok ${ }^{1}$, Ligia Brzezińska-Wcisło ${ }^{1}$
}

\author{
${ }^{1}$ Department of Dermatology, Silesian Medical University, Katowice, Poland \\ Head of the Department: Prof. Ligia Brzezińska-Wcisło MD, PhD \\ 2Institute of Structural Research of Skin, Department of Cosmetology, Silesian Medical University, Sosnowiec, Poland \\ Head of the Department: Krzysztof Jasik PhD
}

Postep Derm Alergol 2015; XXXII (4): 317-319 DOI: 10.5114/pdia.2014.44018

One indication for adalimumab therapy is severe, active Crohn's disease when the response to treatment with a corticosteroid and/or an immunosuppressive agent is insufficient, the treatment is poorly tolerated or there are medical contraindications for this form of therapy [1]. Adverse reactions affecting the skin and mucous membranes during treatment with tumor necrosis factor (TNF) inhibitors are commonly described.

We present the case of a patient in whom severe seborrhoeic dermatitis and a herpes zoster infection developed while using of a TNF inhibitor due to Crohn's disease.

A female patient aged 22. The first skin lesions in the form of erythematous and scaly foci located in the armpits, the groin and the scalp, had appeared 10 months earlier, 5 months after the beginning of treatment with adalimumab due to Crohn's disease. The patient had been under the constant care of a gastroenterologist for 5 years. Azathioprine, prednisone and sulfasalazine had also been used without achieving any significant improvement. During the TNF inhibitor therapy, the skin lesions gradually increased. In addition, in the $12^{\text {th }}$ month of adalimumab use, severe lesions in the form of blisters on an erythematous base with a segmental distribution appeared on the right thigh. The patient was diagnosed with zoster and started on aciclovir at a dose of $5 \times 800 \mathrm{mg} /$ day for 12 days; in addition, adalimumab was discontinued. The lesions caused by herpes zoster disappeared completely, but the eruptions within the scalp, armpits and groin exacerbated further (Figures 1, 2). Laboratory tests showed only slightly increased leucocytosis. Other laboratory tests with arthus-type reactions, general urine test, glucose levels and imaging studies (chest X-ray, ab- dominal ultrasound) were normal. Colonoscopy revealed signs of Crohn's disease. Mycological tests (direct preparation and culture; axillary and inguinal fossae, external acoustic ducts, scalp) were negative. Bacteriological tests (the axillary and inguinal fossae, external acoustic ducts, scalp) - Pseudomonas aeruginosa, Staphylococcus aureus. Histopathological examination (axillary fossa) - thickening of the cornified layer of the epidermis, parakeratosis, spongiosis and slight signs of acanthosis, spinous layer oedema. Perivascular inflammatory infiltrates and dermal oedema. All the findings, which are consistent with the clinical picture, indicate seborrhoeic dermatitis. The patient was administered i.v. ceftriaxone at a dose of $2.0 \mathrm{~g} /$ day for 10 days and antihistamines. External treatment included steroid and antibiotic therapy. When the local symptoms improved, the patient was referred to the Dermatology Outpatient Clinic for follow-up treatment.

Tumor necrosis factor- $\alpha$ (TNF- $\alpha)$ is a cytokine that is at the top of the pro-inflammatory cascade [2], because of which the adverse effects of its inhibitors are well documented. The case that we have described, however, was the first description of severe seborrhoeic dermatitis occurring as a complication of treatment with TNF inhibitors recorded in the literature. Skin lesions appeared as early as 5 months after the beginning of the treatment and gradually exacerbated, and their appearance was clearly associated with starting the medicine. In the literature, there are only cases of paradoxical appearance of psoriasis and psoriasiform lesions (without the characteristics of seborrhoeic dermatitis) in patients treated biologically for other reasons. The mechanism of this phenomenon is probably associated with a possible induction of skin interferon (IFN)- $\alpha$, which predisposes

Address for correspondence: Beata Bergler-Czop, Department of Dermatology, Silesian Medical University, 20/24 Francuska St, 40-027 Katowice, Poland, phone: +48 501352 033, e-mail: bettina2@tlen.pl

Received: 1.01.2014, accepted: 3.04.2014. 


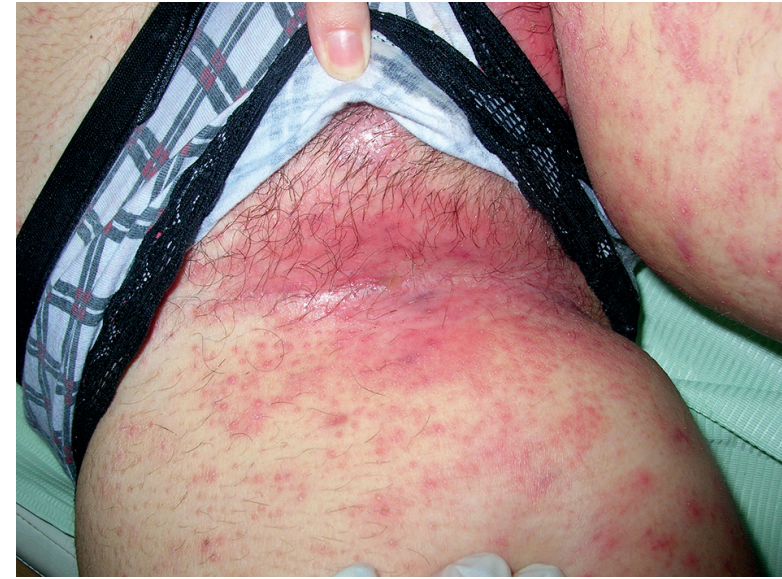

Figure 1. Eruptions in armpits and groin

to the development of psoriatic lesions. Iborra et al. [3] presented a 31-year-old female patient, in whom psoriasis appeared for the first time during the use of adalimumab and infliximab due to Crohn's disease. Doyle et al. [4] described the histopathological characteristics of hair loss induced by TNF inhibitors in 3 patients. In all of them, psoriasis-like signs were observed: acanthosis, parakeratosis with neutrophilic, eosinophilic and plas mocytic infiltrates, as well as subcorneal pustules. These patients had an increased amount of hair in the catagen and telogen phases, follicular miniaturization and perifollicular lymphocytic infiltrates. In 2 patients, there was an improvement after external treatment. Asarch et al. [5] observed 2 patients in whom, during the use of, respectively, adalimumab and infliximab due to psoriasis vulgaris, lichen planus and lichenoid eruptions appeared. Earlier, the authors, analysing literature, had recorded 11 such cases, some of which were psoriasis-like eruptions with histology matching that of lichen planus. It is believed that an impaired balance between TNF- $\alpha$ and INF- $\alpha$ is also responsible for the appearance of these lesions. Sfikakis et al. [6] described 5 patients in whom psoriasis vulgaris appeared for the first time during treatment with TNF inhibitors (adalimumab, etanercept, infliximab). Ko et al. [7] analysed the Medline and PubMed databases in search of adverse skin reactions in the form of psoriatic and psoriasis-like eruptions, occurring during treatment with TNF- $\alpha$ inhibitors. Between 1990 and 2007, 127 such cases were recorded. They were most often caused by infliximab. The lesions appeared, on average, after 10.5 months of therapy. In $40 \%$ of the cases, they were in the form of pustular psoriasis and in 33\% - plaque psoriasis. The most commonly observed complications resulting from treatment with TNF inhibitors include skin and soft tissue infections. In the case we have described, zoster developed during treatment with adalimumab. In addition, Pseudomonas aeruginosa and Staphylococcus aureus were isolated from seborrhoeic

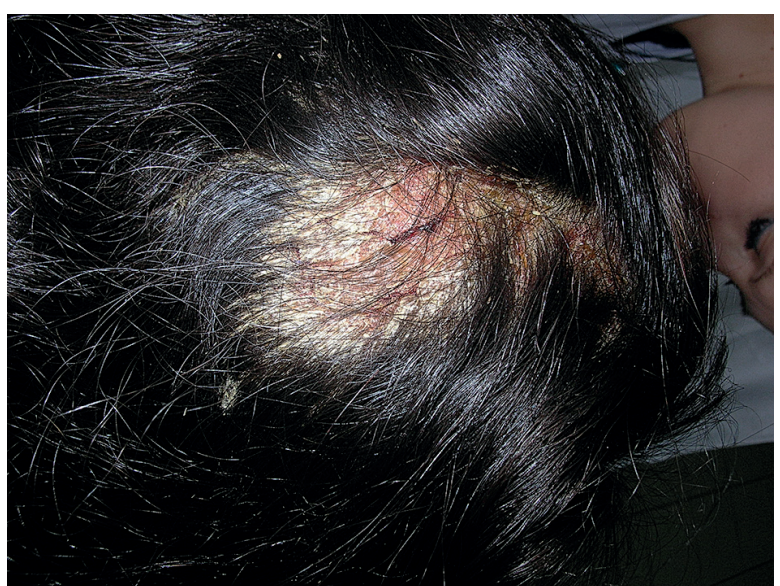

Figure 2. Skin changes on the scalp before treatment

dermatitis lesions. Justice et al. [8] presented a 49-yearold female patient suffering from seronegative rheumatoid arthritis who developed disseminated herpes simplex whilst on infliximab and pustular psoriasis during her 8-month-long treatment with etanercept. A disturbed immunological balance is also responsible for other adverse reactions affecting the skin and mucous membranes observed during biologic therapy. Posada et al. [9] described a 54-year-old woman suffering from Crohn's disease for 30 years, in whom foci of vitiligo appeared after beginning the adalimumab treatment. In some patients, typical drug reactions are described. Marques et al. [10] presented a case of vasculitis in the form of Henoch-Schönlein purpura in the course of adalimumab treatment due to Crohn's disease. The lesions appeared after 18 months of the treatment.

We have presented the case of a patient in whom severe seborrhoeic dermatitis and a herpes zoster infection developed during treatment with a TNF inhibitor due to Crohn's disease. Viral infections are well documented in the literature, but seborrhoeic dermatitis of such severity has been recorded for the first time.

\section{Conflict of interest}

The authors declare no conflict of interest.

\section{References}

1. Serac G, Tubach F, Mariette X, et al. Risk of herpes zoster in patients receiving anti-TNF-alpha in the prospective French RATIO registry. Invest Dermatol 2012; 132: 726-9.

2. Nedoszytko B, Sokołowska-Wojdyło M, RuckemannDziurdzińska K, et al. Chemokines and cytokines network in the pathogenesis of the inflammatory skin diseases: atopic dermatitis, psoriasis and skin mastocytosis. Postep Derm Alergol 2014; 31: 84-91

3. Iborra M, Beltrán B, Bastida G, et al. Infliximab and adalimumab-induced psoriasis in Crohn's disease: a paradoxical side effect. J Crohns Colitis 2011; 5: 157-61. 
4. Doyle LA, Sperling LC, Baksh S, et al. Psoriatic alopecia/alopecia areata-like reactions secondary to anti-tumor necrosis factor-alpha therapy: a novel cause of noncicatricial alopecia. Am J Dermatopathol 2011; 33: 161-6.

5. Asarch A, Gottlieb AB, Lee J, et al. Lichen planus-like eruptions: an emerging side effect of tumor necrosis factor-alpha antagonists. J Am Acad Dermatol 2009; 61: 104-11.

6. Sfikakis PP, Iliopoulos A, Elezoglou A, et al. Psoriasis induced by anti-tumor necrosis factor therapy: a paradoxical adverse reaction. Arthritis Rheum 2005; 52: 2513-8.

7. Ko JM, Gottlieb AB, Kerbleski JF. Induction and exacerbation of psoriasis with TNF-blockade therapy: a review and analysis of 127 cases. J Dermatolog Treat 2009; 20: 100-8.

8. Justice EA, Khan SY, Logan S, Jobanputra P. Disseminated cutaneous Herpes simplex virus-1 in a woman with rheumatoid arthritis receiving infliximab: a case report. J Med Case Rep 2008; 2: 282.

9. Posada C, Flórez A, Batalla A, et al. Vitiligo during treatment of Crohn's disease with adalimumab: adverse effect or cooccurrence? Case Rep Dermatol 2011; 3: 28-31.

10. Marques I, Lagos A, Reis J, et al. Reversible Henoch-Schönlein purpura complicating adalimumab therapy. J Crohns Colitis 2012; 6: 796-9. 